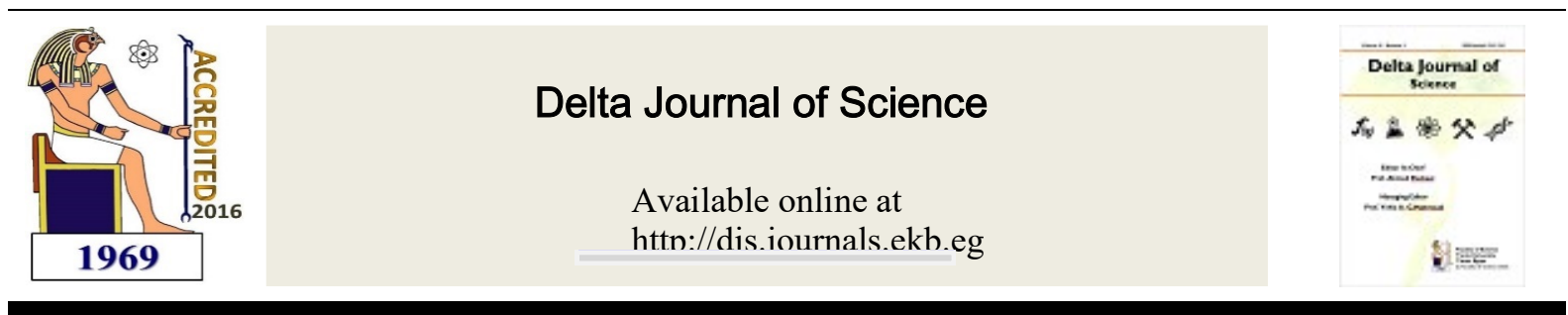

Research Article

MATHEMATICS

\title{
Picard and Homotopy Perturbation Methods for Solving the Time-Fractional Schrödinger Equations
}

E. E. Eladdad and S. M. Aljawazneh

Department of Mathematics, Faculty of Science, Tanta University,Egypt.

${ }^{1}$ e-mail: elsayedeladdad@yahoo.com, ${ }^{2}$ e-mail: Sharhabeelj@yahoo.com

\section{KEY WORDS}

Picard method,

Homotopy

perturbation

method, Time-

fractional;

Schrödinger

equations.

\section{ABSTRACT}

The time-dependent Schrödinger wave equation is the basic partial differential equation of quantum field theory. The study of this equation and its applications play an exceptionally important function in modern physics. From a mathematical point of view, the time-dependent Schrödinger equation is a commutable as mathematics itself. The newest analytical methods to solve linear and nonlinear differential equation is the Homotopy Perturbation Method (HPM) developed to the time-fraction Schrödinger wave equation, which is a combination of homotopy transformation and perturbation. Furthermore, Picard Method (PM) is applied to formulate an approximate iterative solution of the time-fraction Schrödinger equation.

\section{Introduction}

Let's consider the following linear timefractional Schrödinger equation:

$D_{t}^{\alpha} u+i u_{x x}=0, u(x, 0)=f(x)$,

where $i^{2}=-1,0<\alpha \leq 1$, and the non-linear timefractional Schrödinger equation

$$
\begin{aligned}
& i D_{t}^{\alpha} u+\beta u_{x x}+v(x) u+\gamma|u|^{2} u=0, \\
& u(x, 0)=f(x), \quad i^{2}=-1,
\end{aligned}
$$

$$
\left|u^{2}\right|=u \bar{u}, \quad 0<\alpha \leq 1,
$$

where $u(x, t)$ is a complex function, $\beta, \gamma$ are constants, and $v(x)$ is a function in term of $x$.

The linear and non-linear Schrödinger equations are produced in study of the time evolution of the wave function $[1,6,7,8]$, in many of science and engineering sciences and quantum mechanics $[2,17,24]$. 
A large amount of this research was done in previous studies of the linear and nonlinear (timefractional) Schrödinger equation $[2,6,7,11]$.

PM and HPM play an important large role in solving the fractional order differential equations as well as the ordinary order differential equations [9, $13,15,16,18,22,23]$.

Scientists have noted that these are a large number of real-time problems formulated by fractional linear and nonlinear differential equations that are very difficult to process $[10,12,14,20]$. New iterative method has been presented to solve this equation $[3,4,5,19]$.

In this chapter, PM and HPM will be used to approach the linear and nonlinear time-fraction Schrödinger equations, it is well known that these methods can be applied directly to both linear and nonlinear time-fractional Schrödinger equations [2, $6,21]$.

We will circulate the general formula on the linear time-fractional Schrödinger equation, and apply some examples.

The analytical solutions along with the graphs reveal the reliability and full efficiency of the proposed algorithms.

\section{Definitions}

\section{Definition A real function $g(x), x>0$, is} said to be in the space $C_{\mu}, \mu \in R$ if there exists a real number $p(>\mu)$, such that $g(x)=x^{p} g_{1}(x)$, where $g_{1}(x) \in C[0, \infty)$, and it is said to be in the space $C_{\mu}^{\infty}$ if and only if $g^{m} \in C_{\mu}, \mu \geq 1, m \in N$.

Definition The Riemann-Liouville fractional integral operator of order $\alpha>0$, of a function $g \in C_{\mu}, \mu \geq-1$, is defined as:
$I^{\alpha} g(x)=\frac{1}{\Gamma(\alpha)} \int_{0}^{x}(x-t)^{\alpha-1} g(t) d t, \alpha>0, x>0$

$I^{0} g(x)=g(x)$.

Properties of the operator $I^{\alpha}$ can be found in [2], we mention only the following for $g \in C_{\mu}$, $\mu \geq-1, \alpha, \beta \geq 0$ and $\gamma>-1$ :

1- $I^{\alpha} I^{\beta} g(t)=I^{\alpha+\beta} g(t)$,

2- $I^{\alpha} I^{\beta} g(t)=I^{\beta} I^{\alpha} g(t)$,

3- $I^{\alpha} x^{\gamma}=\frac{\Gamma(\gamma+1)}{\Gamma(\alpha+\gamma+1)} x^{\alpha+\gamma}$.

Definition 1.1.3. The fractional derivative of $g(x)$ in the Caputo sense is defined as

$$
\begin{aligned}
D_{t}^{\alpha} g(x)= & I^{m-\alpha} D^{m} g(x) \\
& =\frac{1}{\Gamma(m-\alpha)} \int_{0}^{x}(x-t)^{m-\alpha-1} g^{m}(t) d t,
\end{aligned}
$$

for $m-1<\alpha \geq m, m \in N, x>0, f \in C_{-1}^{m}$.

Also, we need here two of its basic properties.

Lemma 1.1. If $m-1<\alpha \leq m, m \in N$ and $g \in C_{\mu}^{m}$, $\mu \geq-1$, then

$D^{\alpha} I^{\alpha} g(x)=g(x)$,

and

$J^{\alpha} D^{\alpha} g(x)=g(x)-\sum_{k=0}^{m-1} \frac{g^{(k)}\left(0^{+}\right) x^{k}}{k !}, x>0$.

Picard Method (PM).

To illustrate the basic idea of this method, we consider the following general fractional differential equation of arbitrary order $\alpha>0$

$D_{t}^{\alpha}(t)=F\left(t, u^{(k)}(t)\right), m-1<\alpha \leq m, m \in N$,

$\frac{d^{k}}{d t^{k}} u(0)=h_{k}, \quad k=0,1,2, \ldots, m-1$,

where $D_{t}^{\alpha}$ is the fractional differential operator of order $\alpha>0$. In view of the fractional integral 
operators, the initial value fractional problem (5) and (6) is equivalent to the fractional integral equation:

$$
\begin{aligned}
u(t) & =\sum_{k=0}^{m-1} h_{k} \cdot \frac{t^{k}}{k !}+I_{t}^{\alpha}\left[F\left(t, u_{t}^{(k)}(t)\right)\right] \\
& =f+N(u),
\end{aligned}
$$

where $\sum_{k=0}^{m-1} h_{k} \cdot \frac{t^{k}}{k !}, \quad N(u)=I_{t}^{\alpha}\left[F\left(t, u_{t}^{(k)}(t)\right)\right]$ and $I_{t}^{\alpha}$ is the inverse of $D_{t}^{\alpha}$. The required solution $u(t)$ for (7) which is also the solution for (5) and (6) can be obtained as the limit of sequence of function $u_{r+1}(t)$ generated by the recurrence relation:

$$
\left\{\begin{array}{l}
u_{0}=f, \\
u_{r+1}=u_{0}+N\left(u_{r}\right), \quad r=0,1,2, \ldots,
\end{array}\right.
$$

\section{Homotopy Perturbation Method (HMP).}

To illustrate the basic concept of this method, we consider the following differential equation:

$A(u)-g(r)=0, \quad r \in \Omega$,

with boundary conditions:

$B\left(u, \frac{\partial u}{\partial n}\right)=0, \quad r \in \Gamma$,

where $A$ is a general differential operator, $B$ is boundary operator, $g(r)$ is a known analytical function and $\Gamma$ is the boundary of the domain $\Omega$. The operator $A$ can be generally divided into two operator $L$ and $N$, where $L$ is linear and $N$ is a nonlinear. Then equation (9) can be written as follows:

$$
L(u)+N(u)-g(r)=0, \quad r \in \Omega .
$$

Using the Homotopy perturbation technique, we can construct a homotopy $v(r, p): \Omega \times[0,1] \rightarrow R$ which satisfies

$$
\begin{aligned}
H(v, p)= & (1-p)\left[\left(L(v)-L\left(u_{0}\right)\right]+p[L(v)\right. \\
& +N(v)-g(r)]=0,
\end{aligned}
$$

or

$$
\begin{aligned}
H(v, p)= & L(v)-L\left(u_{0}\right)+p L\left(u_{0}\right) \\
& +p[N(v)-g(r)],
\end{aligned}
$$

where $r \in \Omega, p \in[0,1]$ is an embedding parameter and $u_{0}$ is an initial approximation that satisfies the boundary conditions. Now the solution of (12) and (13) can be written as a power series in $p$, as follows:

$v=v_{0}+p v_{1}+p^{2} v_{2}+\ldots$,

and the best approximation solution is:

$u=\lim _{p \rightarrow 1} v=v_{0}+v_{1}+v_{2}+\ldots$.

Now for the general fractional differential equation:

$$
\begin{aligned}
D_{t}^{\alpha}(x, t)= & L\left(u, u_{x}, u_{x x}\right)+N\left(u, u_{x}, u_{x x}\right)+g(x, t), \\
& t>0,
\end{aligned}
$$

where $L$ is a liner operator, $N$ is a nonlinear operator, $g(x, t)$ is a known analytic function and $D^{\alpha}$, is the Caputo fractional derivative of order $\alpha$, where $m-1<\alpha \leq m$, subject to the initial conditions:

$u^{(k)}(x, 0)=h_{k}(x), k=0,1,2,3, \ldots, m-1$.

In view of the homotopy technique, we can construct the following homotopy:

$D_{t}^{\alpha} u-L\left(u, u_{x}, u_{x x}\right)-g(x, t)=p\left[N\left(u, u_{x}, u_{x x}\right)\right]$,

or

$$
D_{t}^{\alpha} u-g(x, t)=p\left[\left(u, u_{x}, u_{x x}\right)+N\left(u, u_{x}, u_{x x}\right)\right] .
$$

The basic assumption is that the solution of (19) can be written as a power series in $p$ :

$u=u_{0}+p u_{1}+p^{2} u_{2}+\ldots$.

Finally, the $n$-term approximate solution is 
$u(x)=\sum_{i=0}^{n-1} u_{i}$

\section{Application}

\section{Linear Time-Fractional Schrödinger}

\section{Equation.}

Let's consider the linear time-fractional Schrödinger equation of the following form

$D_{t}^{\alpha} u \pm i u_{x x}=0, \quad u(x, 0)=f(x), 0<\alpha \leq 1$

To solve the linear time-fractional Schrödinger equation (22) by using:

1- PM: We proceed as follows:

$u_{j+1}=u_{0} \mp I_{t}^{\alpha} i\left[\left(u_{j}\right)_{x x}\right], \quad j \geq 0, \quad 0<\alpha \leq 1, \quad(23)$

$u_{0}=f(x)$,

$u_{1}=f(x) \mp I_{t}^{\alpha} i\left[\frac{\partial^{2} f}{\partial x^{2}}\right]=f(x) \mp i \frac{\partial^{2} f}{\partial x^{2}} \frac{t^{\alpha}}{\Gamma(\alpha+1)}$,

$u_{2}=f(x) \mp I_{t}^{\alpha} i\left[f(x) \mp i \frac{\partial^{2} f}{\partial x^{2}} \frac{t^{\alpha}}{\Gamma(\alpha+1)}\right]_{x x}$

$=f(x) \pm i \frac{\partial^{2} f}{\partial x^{2}} \frac{t^{\alpha}}{\Gamma(\alpha+1)}+i^{2} \frac{\partial^{4} f}{\partial x^{4}} \frac{t^{2 \alpha}}{\Gamma(2 \alpha+1)}$,

$u_{3}=f(x) \mp I_{t}^{\alpha} i\left[f(x) \mp i \frac{\partial^{2} f}{\partial x^{2}} \frac{t^{\alpha}}{\Gamma(\alpha+1)}\right.$

$\left.+i^{2} \frac{\partial^{4} f}{\partial x^{4}} \frac{t^{2 \alpha}}{\Gamma(2 \alpha+1)}\right]$

$=f(x) \mp i \frac{\partial^{2} f}{\partial x^{2}} \frac{t^{\alpha}}{\Gamma(\alpha+1)}+i^{2} \frac{\partial^{4} f}{\partial x^{4}} \frac{t^{2 \alpha}}{\Gamma(2 \alpha+1)}$

$\mp i^{3} \frac{\partial^{6} f}{\partial x^{6}} \frac{t^{3 \alpha}}{\Gamma(3 \alpha+1)}$

For higher order terms, we can obtain:

$u(x, t)=f(x)+\sum_{n=1}^{\infty}(\mp 1)^{n}(i)^{n} \frac{t^{n \alpha}}{\Gamma(n \alpha+1)} \frac{\partial^{2 n} f}{\partial x^{2 n}}$.

For the special case $\alpha=1$, we obtain from equation

$u(x, t)=f(x)+\sum_{n=1}^{\infty}(\mp 1)^{n} \frac{(i t)^{n}}{n !} \frac{\partial^{2 n} f(x)}{\partial x^{2 n}}$.
2-HPM: we suppose that the solution of the problem can be written as a power series in $p$ :

$u=u_{0}+p u_{1}+p^{2} u_{2}+\ldots$,

and let us construct the homotopy:

$D_{t}^{\alpha}=\mp p\left[i u_{x x}\right]$,

Substitute (27) in (28), the homotopy for (22) becomes:

$$
\begin{aligned}
D_{t}^{\alpha}\left[u_{0}+p u_{1}+p^{2} u_{2}+p^{3} u_{3}+\ldots\right]= & \mp p i\left[u_{0}+p u_{1}+p^{2} u_{2}\right. \\
& \left.+p^{3} u_{3}+\ldots\right]_{x x},
\end{aligned}
$$

Equating the coefficients of equal power of $p$ in both sides of (29), we obtain:

$$
\begin{array}{ll}
p^{0}: D_{t}^{\alpha} u_{0}=0, & u_{0}(x, 0)=f(x), \\
p^{1}: D_{t}^{\alpha} u_{1}=\mp i u_{0_{x x}}, & u_{1}(x, 0)=0 \\
p^{2}: D_{t}^{\alpha} u_{2}=\mp i u_{1_{x x}}, & u_{2}(x, 0)=0 \\
p^{3}: D_{t}^{\alpha} u_{3}=\mp i u_{2_{x x}}, & u_{3}(x, 0)=0 \\
\vdots &
\end{array}
$$

Solving the above set of equations (30), we get the first terms of the homotopy perturbation solution for (22):

$$
\begin{aligned}
& u_{0}=f(x) \\
& u_{1}=\mp i \frac{\partial^{2} f}{\partial x^{2}} \frac{t^{\alpha}}{\Gamma(\alpha+1)} \\
& u_{2}=+i^{2} \frac{\partial^{4} f}{\partial x^{4}} \frac{t^{2 \alpha}}{\Gamma(2 \alpha+1)} \\
& u_{3}=\mp i^{3} \frac{\partial^{6} f}{\partial x^{6}} \frac{t^{3 \alpha}}{\Gamma(3 \alpha+1)}
\end{aligned}
$$

So the solution will be

$$
\begin{aligned}
& u(x, t)=u_{0}+u_{1}+u_{2}+u_{3}+\ldots \\
& =f(x) \mp i \frac{\partial^{2} f}{\partial x^{2}} \frac{t^{\alpha}}{\Gamma(\alpha+1)}+i^{2} \frac{\partial^{4} f}{\partial x^{4}} \frac{t^{2 \alpha}}{\Gamma(2 \alpha+1)} \\
& \mp i^{3} \frac{\partial^{6} f}{\partial x^{6}} \frac{t^{3 \alpha}}{\Gamma(3 \alpha+1)}+\ldots .
\end{aligned}
$$


For higher terms we get

$u(x, t)=f(x)+\sum_{n=1}^{\infty}(\mp 1)^{n}(i)^{n} \frac{t^{n \alpha}}{\Gamma(n \alpha+1)} \frac{\partial^{2 n} f(x)}{\partial x^{2 n}}$.

For special case $\alpha=1$, we obtain from equation

$u(x, t)=f(x)+\sum_{n=1}^{\infty}(\mp 1)^{n} \frac{(i t)^{n}}{n !} \frac{\partial^{2 n} f(x)}{\partial x^{2 n}}$.

Example 3.1. Consider the linear time-fractional Schrödinger equation

$D_{t}^{\alpha} u_{x}+i u_{x x}=0, \quad 0<\alpha \leq 1$,

with the initial condition $u(x, 0)=k e^{i m x}$.

\section{1- By PM:}

We construct the following iteration formula for Picard:

$u_{j+1}=u_{0}-I_{t}^{\alpha} i\left[\left(u_{j}\right)_{x x}\right], \quad j \geq 0$.

Therefore, we can obtain the following first few terms of the required solution.

$$
\begin{aligned}
u_{0}= & k e^{i m x}, \\
u_{1}= & k^{i m x}+i k m^{2} \frac{t^{\alpha}}{\Gamma(\alpha+1)}, \\
u_{2}= & k e^{i m x}+i k m^{2} e^{i m x} \frac{t^{\alpha}}{\Gamma(\alpha+1)} \\
& +i^{2} k m^{4} e^{i m x} \frac{t^{2 \alpha}}{\Gamma(2 \alpha+1)}, \\
u_{3}= & k e^{i m x}+i k m^{2} e^{i m x} \frac{t^{\alpha}}{\Gamma(\alpha+1)} \\
+i^{2} k m^{4} e^{i m x} & \frac{t^{2 \alpha}}{\Gamma(2 \alpha+1)}+i^{3} k m^{6} e^{i m x} \frac{t^{3 \alpha}}{\Gamma(3 \alpha+1)},
\end{aligned}
$$

then

$$
\begin{gathered}
u=k e^{i m x}\left(1+i m^{2} \frac{t^{\alpha}}{\Gamma(\alpha+1)}+i^{2} m^{4} \frac{t^{2 \alpha}}{\Gamma(2 \alpha+1)}\right. \\
\left.+i^{3} m^{6} \frac{t^{3 \alpha}}{\Gamma(3 \alpha+1)}\right) .
\end{gathered}
$$

In closed form, we obtain the solution

$$
u=k e^{i m x}\left(\sum_{n=0}^{\infty}\left(i m^{2}\right)^{n} \frac{t^{n \alpha}}{\Gamma(n \alpha+1)}\right) .
$$

For the special case $\alpha=1$, we obtain that:

$$
\begin{aligned}
u & =k e^{i m x}\left(1+i m^{2} t+\frac{\left(i m^{2} t\right)^{2}}{2 !}+\frac{\left(i m^{2} t\right)^{3}}{3 !}+\ldots\right) \\
& =k e^{i m(x+m t)}
\end{aligned}
$$

which is an exact solution for (34).

The same result can be obtained by applying the general formula (25) for solving (4.34) in the form:

$$
\begin{aligned}
u= & k e^{i m x}+i k m^{2} e^{i m x} \frac{t^{\alpha}}{\Gamma(\alpha+1)} \\
& +i^{2} k m^{4} e^{i m x} \frac{t^{2 \alpha}}{\Gamma(2 \alpha+1)} \\
& +i^{3} k m^{6} e^{i m x} \frac{t^{3 \alpha}}{\Gamma(3 \alpha+1)}+\ldots,
\end{aligned}
$$

then

$$
\begin{gathered}
u=k e^{i m x}\left(1+i m^{2} \frac{t^{\alpha}}{\Gamma(\alpha+1)}+i^{2} m^{4} \frac{t^{2 \alpha}}{\Gamma(2 \alpha+1)}\right. \\
\left.+i^{3} m^{6} \frac{t^{3 \alpha}}{\Gamma(3 \alpha+1)}\right) .
\end{gathered}
$$

In closed form, we obtain:

$$
u=k e^{i m x}\left(\sum_{n=0}^{\infty}\left(i m^{2}\right)^{n} \frac{t^{n \alpha}}{\Gamma(n \alpha+1)}\right) .
$$

For the special case $\alpha=1$, we obtain that

$$
\begin{aligned}
u & =k e^{i m x}\left(1+i m^{2} t+\frac{\left(i m^{2} t\right)^{2}}{2 !}+\frac{\left(i m^{2} t\right)^{3}}{3 !}+\ldots\right) \\
& =k e^{i m(x+m t)}
\end{aligned}
$$

which is the exact solution for (34).

2- By HPM: The homotopy for (34) becomes

$D_{t}^{\alpha} u=-i p\left[u_{x x}\right]$,

we suppose that the solution of the problem (34) is in the form:

$u=u_{0}+p u_{1}+p^{2} u_{2}+p^{3} u_{3}+\ldots$. 
Substituting (40) into (39), we obtain:

$$
\begin{aligned}
D_{t}^{\alpha}\left[u_{0}\right. & \left.+p u_{1}+p^{2} u_{2}+p^{3} u_{3}+\ldots\right]=-i p\left[u_{0}+p u_{1}\right. \\
& \left.+p^{2} u_{2}+p^{3} u_{3}+\ldots\right]_{x x}
\end{aligned}
$$

Equating the terms of equal powers of $p$ in both sides of the above equation, we obtain:

$$
\left.\begin{array}{ll}
p^{0}: D_{t}^{\alpha} u_{0}=0, & u_{0}(x, 0)=k e^{i m x}, \\
p^{1}: D_{t}^{\alpha} u_{1}=-i u_{0_{x x}}, & u_{1}(x, 0)=0, \\
p^{2}: D_{t}^{\alpha} u_{2}=-i u_{1_{x x}}, & u_{2}(x, 0)=0, \\
p^{3}: D_{t}^{\alpha} u_{3}=-i u_{2_{x x}}, & u_{3}(x, 0)=0 \\
\vdots &
\end{array}\right\}
$$

Solving the above set equations in (42), we obtain:

$$
\begin{aligned}
& u_{0}=k e^{i m x}, \\
& u_{1}=i k m^{2} e^{i m x} \frac{t^{\alpha}}{\Gamma(\alpha+1)}, \\
& u_{2}=i^{2} k m^{4} e^{i m x} \frac{t^{2 \alpha}}{\Gamma(2 \alpha+1)}, \\
& u_{3}=i^{3} k m^{6} e^{i m x} \frac{t^{3 \alpha}}{\Gamma(3 \alpha+1)},
\end{aligned}
$$

So the solution will be

$$
\begin{aligned}
& u(x, t)=u_{0}+u_{1}+u_{2}+u_{3}+\ldots \\
& =k e^{i m x}+i k m^{2} e^{i m x} \frac{t^{\alpha}}{\Gamma(\alpha+1)} \\
& +i^{2} k m^{4} e^{i m x} \frac{t^{2 \alpha}}{\Gamma(2 \alpha+1)}+i^{3} k m^{6} e^{i m x} \frac{t^{3 \alpha}}{\Gamma(3 \alpha+1)}+\ldots,
\end{aligned}
$$

then

$$
\begin{aligned}
u= & \sum_{i=0}^{3} u_{i}=k e^{i m x}\left(1+i m^{2} \frac{t^{\alpha}}{\Gamma(\alpha+1)}+i^{2} m^{4} \frac{t^{2 \alpha}}{\Gamma(2 \alpha+1)}\right. \\
& +i^{3} m^{6} \frac{t^{3 \alpha}}{\Gamma(3 \alpha+1)} .
\end{aligned}
$$

In closed form, we obtain the solution

$$
u=k e^{i m x}\left(\sum_{n=0}^{\infty}\left(i m^{2}\right)^{n} \frac{t^{n \alpha}}{\Gamma(n \alpha+1)}\right) .
$$

For the special cases $\alpha=1$, we obtain

$$
\begin{aligned}
u(x, t)= & k e^{i m x}+i k m^{2} e^{i m x} t+i^{2} k m^{4} e^{i m x} \frac{t^{2}}{2 !} \\
& +i^{3} k m^{6} e^{i m x} \frac{t^{3}}{3 !}+\ldots=k e^{i m(x+m t)},
\end{aligned}
$$

which is the same exact solution as obtained by PM.

It is clear that the result obtained by HPM in (45) is the same result as obtained by PM (36).

In Fig. (3), we have Plotted the approximate solution for the linear Schrödinger equation (34) for the closed a) $\alpha=0.25$, b) $\alpha=0.5$, c) $\alpha=0.75$, d) $\alpha=1$, and e) the corresponding exact solution at $m=k=1$.

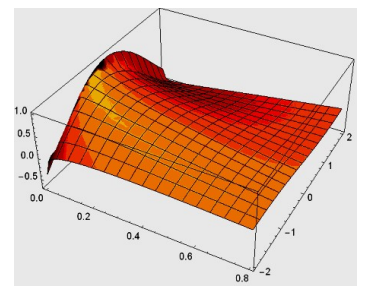

(a) $\alpha=0.25$

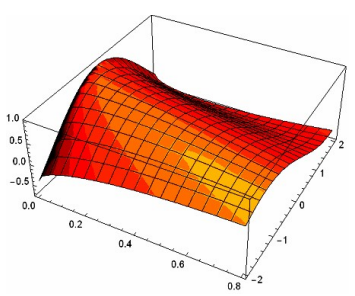

(c) $\alpha=0.75$

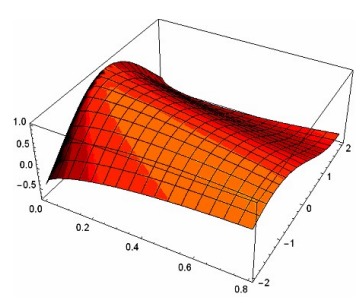

(b) $\alpha=0.50$

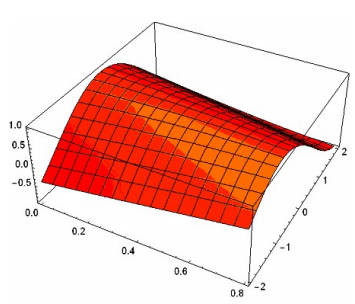

(d) $\alpha=1.0$

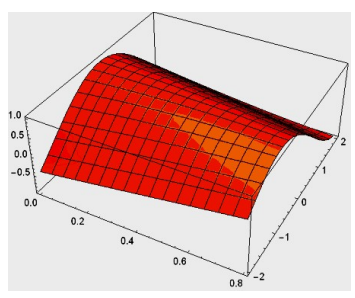

(e) Exact solution

Fig. 3. The surfaces show the approximate solution for (34) for various values of $\alpha$ with the corresponding exact solution at $m=k=1$. 
Example 3.2. Consider the linear time-fractional Schrödinger equation.

$D_{t}^{\alpha} u+i u_{x x}=0$,

with the initial condition

$u(x, 0)=1+\cosh 2 x$.

1- By PM: using formula (25) lead to the following result:

$$
\begin{aligned}
u(x, t)= & 1+\cosh 2 x-4 i \cosh 2 x \frac{t^{\alpha}}{\Gamma(\alpha+1)} \\
& +16 i^{2} \cosh 2 x \frac{t^{2 \alpha}}{\Gamma(2 \alpha+1)} \\
& -64 i^{3} \cosh 2 x \frac{t^{3 \alpha}}{\Gamma(3 \alpha+1)} \\
= & 1+\cosh 2 x\left(1-4 i \frac{t^{\alpha}}{\Gamma(\alpha+1)}+16 i^{2} \frac{t^{2 \alpha}}{\Gamma(2 \alpha+1)}\right. \\
& -64 i \frac{t^{3 \alpha}}{\Gamma(3 \alpha+1)}+\ldots,
\end{aligned}
$$

then

$$
u(x, t)=1+\cosh 2 x\left(\sum_{n=0}^{\infty}(-4 i)^{n} \frac{t^{n \alpha}}{\Gamma(n \alpha+1)}\right) .
$$

For the special case $\alpha=1$, we obtain

$u(x, t)=1+\cosh 2 x-4 i \cosh 2 x t+16 i^{2} \cosh 2 x \frac{t^{2}}{2 !}$

$$
-64 i^{3} \cosh 2 x \frac{t^{3}}{3 !}+\ldots=1+e^{-4 i t} \cosh 2 x,
$$

which is the exact solution for (47).

2- By HPM: by using formula (33), we obtain that:

$$
\begin{aligned}
& u_{0}=1+\cos 2 x, \\
& u_{1}=-4 i \cosh 2 x \frac{t^{\alpha}}{\Gamma(\alpha+1)}, \\
& u_{2}=16 i^{2} \cosh 2 x \frac{t^{2 \alpha}}{\Gamma(2 \alpha+1)}, \\
& u_{3}=-64 i^{3} \cosh 2 x \frac{t^{3 \alpha}}{\Gamma(2 \alpha+1)},
\end{aligned}
$$

So the solution will be:

$$
\begin{aligned}
u(x, t)= & u_{0}+u_{1}+u_{2}+u_{3}+\ldots \\
= & 1+\cosh 2 x-4 i \cosh 2 x \frac{t^{\alpha}}{\Gamma(\alpha+1)} \\
& +16 i^{2} \cosh 2 x \frac{t^{2 \alpha}}{\Gamma(2 \alpha+1)} \\
& -64 i^{3} \cosh 2 x \frac{t^{3 \alpha}}{\Gamma(2 \alpha+1)}+\ldots \\
= & 1+\cosh 2 x\left(1-4 i \frac{t^{\alpha}}{\Gamma(\alpha+1)}+16 i^{2} \frac{t^{2 \alpha}}{\Gamma(2 \alpha+1)}\right. \\
& \left.-64 i^{3} \frac{t^{3 \alpha}}{\Gamma(3 \alpha+1)}+\ldots\right)
\end{aligned}
$$

then

$$
u(x, t)=1+\cosh 2 x\left(\sum_{n=0}^{\infty}(-4 i)^{n} \frac{t^{n \alpha}}{\Gamma(n \alpha+1)}\right)
$$

For the special case $\alpha=1$, we obtain that

$$
\begin{aligned}
u(x, t)= & 1+\cosh 2 x-4 i \cosh 2 x t+16 i^{2} \cosh 2 x \frac{t^{2}}{2 !} \\
& -64 i^{3} \cosh 2 x \frac{t^{3}}{3 !}+\ldots \\
= & 1+e^{-4 i t} \cosh 2 x .
\end{aligned}
$$

which is an exact solution for (47).

It is clear that the result obtained by HPM in (52) is the same result as obtained by PM (49).

In Fig. (2), we have Plotted the approximate solution for the linear Schrödinger equation (47) for the case a) $\alpha=0.25$, b) $\alpha=0.5$, c) $\alpha=0.75$, d) $\alpha=1$, and e) the corresponding exact solution. 


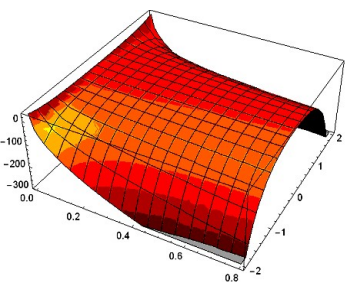

(a) $\alpha=0.25$

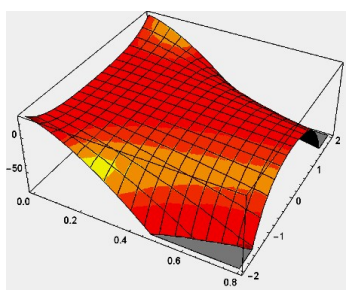

(c) $\alpha=0.75$

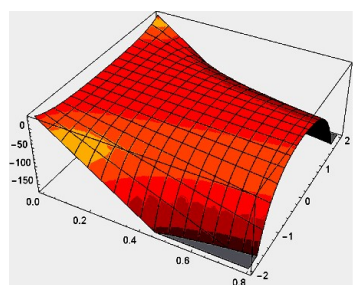

(b) $\alpha=0.50$

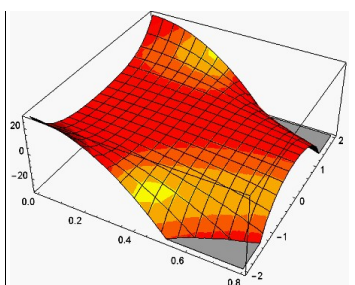

(d) $\alpha=1.0$

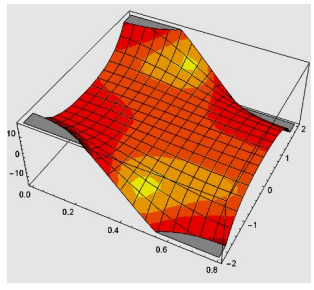

(e) Exact solution

Fig. 3. The approximate solution for (47) for various values at $\alpha$ with corresponding exact solution.

\section{Non Linear Time-Fractional Schrödinger}

Equation.

Consider the following nonlinear

Schrödinger equation in the following form

$i D_{t}^{\alpha} u+\beta u_{x x}+v(x) u+\gamma|u|^{2} u=0,0<\alpha \leq 1$,

which the initial conditions:

$u(x, 0)=f(x)$.

We can rewrite the equation (53) in the form $D_{t}^{\alpha} u-B i u_{x x}-i v(x) u-\gamma i u^{2} \bar{u}=0$ where $\bar{u}$ is the complex conjugate of $u,\left|u^{2}\right|=u \bar{u}$ and $\beta, \gamma$ are constants, $u(x, t)$ is a complex function, $v(x)$ is a function with $x$.

1- By PM: we proceed as follows

$$
\begin{gathered}
u_{j+1}=u_{0}+I_{t}^{\alpha}\left[i \beta u_{j_{x x}}+i v(x) u_{j}+i \gamma u_{j}^{2} \bar{u}_{j}\right], \\
j \geq 0,0<\alpha \leq 1
\end{gathered}
$$

$$
\begin{aligned}
& u_{0}=f(x), \\
& u_{1}=f(x)+\left[i \gamma \overline{u_{0}} u_{0}^{2}+i u_{0} v(x)+i \beta u_{0_{x x}}\right] \frac{t^{\alpha}}{\Gamma(\alpha+1)}, \\
& u_{2}=f(x)+\left[i \gamma \bar{u}_{0} u_{0}^{2}+i u_{0} v(x)+i \beta u_{0_{x x}}\right] \frac{t^{\alpha}}{\Gamma(\alpha+1)}
\end{aligned}
$$

$$
\begin{aligned}
& +\left[-2 \gamma^{2} u_{0}^{2} u_{0}^{3}-3 \gamma \bar{u}_{0} u_{0}^{2} v(x)-u_{0} v^{2}(x)\right. \\
& -2 \beta \gamma \bar{u}_{0} u_{0_{x}}^{2}-4 \beta \gamma u_{0} \bar{u}_{0_{x}} u_{0_{x}}^{2}-\beta \gamma u_{0}^{2} u_{0_{x}}^{2} \bar{u}_{0_{x x}} \\
& -4 \beta \gamma \bar{u}_{0} u_{0} u_{0_{x x}}-2 \beta v(x) u_{0_{x x}}-\beta \gamma u_{0}^{2} \bar{u}_{0_{x}} u_{0_{x x}} \\
& \left.-\beta^{2} u_{0_{x x x x}}-2 \beta u_{0_{x}} v^{\prime}(x)-\beta u_{0} v^{\prime \prime}(x)\right]
\end{aligned}
$$

$$
\begin{aligned}
& \frac{t^{2 \alpha}}{\Gamma(2 \alpha+1)}+\ldots, \\
u_{3}= & f(x)+\left[i \gamma u_{0}^{2} \bar{u}_{0}+i u_{0} v(x)+i \beta u_{0_{x x}}\right] \frac{t^{\alpha}}{\Gamma(\alpha+1)} \\
& +\left[-2 \gamma^{2} u_{0}^{2} u_{0}^{3}-3 \gamma u_{0}^{2} \bar{u}_{0} v(x)-u_{0} v^{2}(x)\right. \\
& -2 \beta \gamma \bar{u}_{0} u_{0_{x}}^{2}-4 \beta \gamma u_{0} \bar{u}_{0_{x}} u_{0_{x}}^{2}-\beta \gamma u_{0}^{2} u_{0_{x}}^{2} \bar{u}_{0_{x x}} \\
& -4 \beta \gamma \bar{u}_{0} u_{0} u_{0_{x x}}-2 \beta v(x) u_{0_{x x}}-\beta \gamma u_{0}^{2} \bar{u}_{0_{x}} u_{0_{x x}} \\
& \left.-\beta^{2} u_{0_{x x x}}-2 \beta u_{0_{x}} v^{\prime}(x)-\beta u_{0} v^{\prime \prime}(x)\right] \frac{t^{2 \alpha}}{\Gamma(2 \alpha+1)} \\
& +\left[i \gamma^{3} \bar{u}_{0}^{3} u_{0}^{4}-2 i \gamma^{2} \bar{u}_{0}^{2} u_{0}^{3} v(x)-i \gamma \bar{u}_{0} u_{0}^{2} v^{2}(x)\right. \\
& -2 i \beta \gamma^{2} \bar{u}_{0}^{2} u_{0}^{2} u_{0_{x x}}-2 i \beta \gamma \bar{u}_{0} u_{0} r(x) u_{0_{x x}} \\
& \left.-i \beta^{2} \gamma \bar{u}_{0} u_{0_{x x}}^{2}\right] \frac{t^{3 \alpha}}{\Gamma(3 \alpha+1)}+\ldots
\end{aligned}
$$

2- By HPM: we suppose that the solution of the problem can written as a power series in $p$

$$
\begin{aligned}
& D_{t}^{\alpha} u=i p\left[\beta u_{x x}+v(x) u+\gamma u^{2} \bar{u}\right], \\
& u=u_{0}+p u_{1}+p^{2} u_{2}+p^{3} u_{3}+\ldots
\end{aligned}
$$

Substituting (56) in (53), the homotopy for (53) becomes

$$
\begin{aligned}
D_{t}^{\alpha} & {\left[u_{0}+p u_{1}+p^{2} u_{2}+p^{3} u_{3}+\ldots\right]=i p\left[\beta \left[u_{0}+p_{1} u_{1}\right.\right.} \\
& \left.+p^{2} u_{2}+p^{3} u_{3}+\ldots\right]_{x x}+v(x)\left[u_{0}+p u_{1}+p^{2} u_{2}+p^{3} u_{3}\right. \\
& +\ldots]+\gamma\left[u_{0}+p u_{1}+p^{2} u_{2}+p^{3} u_{3}+\ldots\right]^{2}\left[\bar{u}_{0}+p \bar{u}_{1}\right. \\
& \left.\left.+p^{2} \bar{u}_{2}+p^{3} \bar{u}_{3}\right]\right]
\end{aligned}
$$


Equating the coefficient of equal power of $p$ in both sides of equation (57), we obtain

$$
\begin{aligned}
& p^{0}: D_{t}^{\alpha} u_{0}=0, \quad u_{0}(x, 0)=f(x), \\
& p^{1}: D_{t}^{\alpha} u_{1}=\beta i u_{0_{x x}}+i v(x) u_{0_{x}}+\gamma i u_{0}^{2} \bar{u}_{0}, u_{1}(x, 0)=0, \\
& p^{2}: D_{t}^{\alpha} u_{2}=\beta i u_{1_{x x}}+i v(x) u_{1_{x}}+\gamma i u_{0}^{2} \bar{u}_{1} \\
& +2 \gamma i u_{0} u_{1} \bar{u}_{0}, \quad u_{2}(x, 0)=0, \\
& p^{3}: D_{t}^{\alpha} u_{3}=\beta i u_{2_{x x}}+i v(x) u_{2_{x}}+\gamma i u_{0}^{2} \bar{u}_{2} \\
& +\gamma i \bar{u}_{0}\left(u_{1}^{2}+2 u_{0} u_{2}\right)+2 \gamma i u_{0} u_{1} \bar{u}_{1}, u_{3}(x, 0)=0, \\
& \vdots
\end{aligned}
$$

Solving the above set of equation in (58), we get the first terms of the homotopy perturbation

$$
\begin{aligned}
u_{0}= & f(x), \\
u_{1}= & {\left[\beta i u_{0_{x x}}+i v(x) u_{0_{x}}+\gamma i u_{0}^{2} \overline{u_{0}}\right] \frac{t^{\alpha}}{\Gamma(\alpha+1)}, } \\
u_{2}= & {\left[\beta i u_{1_{x x}}+i v(x) u_{1_{x}}+\gamma i u_{0}^{2} \overline{u_{1}}+2 \gamma i u_{0} u_{1} \bar{u}_{0}\right] } \\
& \frac{t^{2 \alpha}}{\Gamma(2 \alpha+1)}, \\
u_{3}= & {\left[\beta i u_{2_{x x}}+i v(x) u_{2_{x}}+\gamma i u_{0}^{2} \overline{u_{2}}+\gamma i \bar{u}_{0}\left(u_{1}^{2}\right.\right.} \\
& \left.\left.+2 u_{0} u_{2}\right)+2 \gamma i u_{0} u_{1} \bar{u}_{1}\right] \frac{t^{3 \alpha}}{\Gamma(3 \alpha+1)},
\end{aligned}
$$

$\vdots$

So the solution will be $u(x, t)=u_{0}+u_{1}+u_{2}+u_{3}+\ldots$.

Example 3. Consider the following cubic nonlinear time-fractional Schrödinger equation

$i D_{t}^{\alpha} u+\beta u_{x x}-2|u|^{2} u=0$,

with the initial condition

$u(x, 0)=e^{i x}$.

The exact solution is $u(x, t)=e^{i(x-3 t)}$. We have $\beta=1, v(x)=0, \quad \gamma=-2$, we can rewrite the equation (59) in the form

$D_{t}^{\alpha}-i u_{x x}+2 i u^{2} \bar{u}=0$.
1- By PM: we constract the following iteration formula for Picard:

$u_{j+1}=u_{0}+I_{t}^{\alpha}\left[i u_{j_{x x}}-2 i u_{j}^{2} u_{j}\right], \quad j \geq 0$,

therefore, we can obtain the following first few terms of the required solution

$$
\begin{aligned}
& u_{0}=e^{i x}, \\
& u_{1}=e^{i x}-3 i e^{i x} \frac{t^{\alpha}}{\Gamma(\alpha+1)}, \\
& u_{2}=e^{i x}-3 i e^{i x} \frac{t^{\alpha}}{\Gamma(\alpha+1)}+9 i^{2} e^{i x} \frac{t^{2 \alpha}}{\Gamma(2 \alpha+1)}, \\
& u_{3}=e^{i x}-3 i e^{i x} \frac{t^{\alpha}}{\Gamma(\alpha+1)}+3^{2} e^{i x} i^{2} \frac{t^{2 \alpha}}{\Gamma(2 \alpha+1)} \\
& \vdots \\
& \text { then } 3^{3} i^{3} e^{i x} \frac{t^{3 \alpha}}{\Gamma(3 \alpha+1)}, \\
& u=e^{i x}\left(1-3 i \frac{t^{\alpha}}{\Gamma(\alpha+1)}+\frac{(3 i)^{2} t^{2 \alpha}}{\Gamma(2 \alpha+1)}-\frac{(3 i)^{3} t^{3 \alpha}}{\Gamma(3 \alpha+1)}+\ldots\right) .
\end{aligned}
$$

In closed form, we obtain the solution

$u=e^{i x}\left(\sum_{n=0}^{\infty}(-3 i)^{n} \frac{t^{n \alpha}}{\Gamma(n \alpha+1)}\right)$.

For the special case $\alpha=1$, we obtain

$u(x, t)=e^{i x}\left(1-3 i t+\frac{(3 i t)^{2}}{2 !}-\frac{(3 i t)^{3}}{3 !}+\ldots\right)$,

which is an exact solution for (59).

2- By HPM: the homotopy (59) becomes

$D_{t}^{\alpha} u=i p\left[u_{x x}-2 u^{2} \bar{u}\right]$,

we suppose that the solution of the problem (64) is in the form

$u=u_{0}+p u_{1}+p^{2} u_{2}+p^{3} u_{3}+\ldots$.

Substituting (65) into (64), we obtain:

$$
\begin{gathered}
D_{t}^{\alpha}\left[u_{0}+p u_{1}+p^{2} u_{2}+p^{3} u_{3}+\ldots\right]=i p\left[\left[u_{0}+p u_{1}+p^{2} u_{2}\right.\right. \\
\left.+p^{3} u_{3}+\ldots\right]_{x x}-2\left[u_{0}+p u_{1}+p^{2} u_{2}+p^{3} u_{3}+\ldots\right]^{2} \\
\left.\left[\bar{u}_{0}+p \bar{u}_{1}+p^{2} \bar{u}_{2}+p^{3} \bar{u}_{3}+\ldots\right]\right]
\end{gathered}
$$


Equating the terms of equal powers $p$ in both sides of the above equation we obtain:

$$
\begin{aligned}
& p^{0}: D_{t}^{\alpha} u_{0}=0 \\
& p^{1}: D_{t}^{\alpha} u_{1}=i u_{0_{x x}}-2 i u_{0}^{2} \bar{u}_{0}, \quad u_{1}(x, 0)=0 \text {, } \\
& p^{2}: D_{t}^{\alpha} u_{2}=i u_{1_{x x}}-2 i u_{0}^{2} \bar{u}_{1}-4 i u_{0} u_{1} \bar{u}_{0}, u_{2}(x, 0)=0 \text {, } \\
& p^{3}: D_{t}^{\alpha} u_{3}=i u_{2_{x x}}-2 i u_{0}^{2} \bar{u}_{2}-2 i \bar{u}_{0}\left(u_{1}^{2}+2 u_{0} u_{2}\right) \\
& -4 i u_{0} u_{1} \bar{u}_{1}, \quad u_{2}(x, 0)=0, \\
& \text { : }
\end{aligned}
$$

Solving the above set of equation in (67), we obtain $u_{0}=e^{i x}$,

$$
u_{1}=-3 i e^{i x} \frac{t^{\alpha}}{\Gamma(\alpha+1)},
$$$$
u_{2}=9 i^{2} e^{i x} \frac{t^{2 \alpha}}{\Gamma(2 \alpha+1)} \text {, }
$$$$
u_{3}=-63 i^{3} e^{i x} \frac{t^{3 \alpha}}{\Gamma(3 \alpha+1)}+18 i^{3} e^{i x} \frac{t^{3 \alpha}}{\Gamma(3 \alpha+1)} \frac{\Gamma(2 \alpha+1)}{\Gamma(\alpha+1)^{2}} .
$$

So the solution will be

$$
\begin{aligned}
u(x, t)= & u_{0}+u_{1}+u_{2}+u_{3}+\ldots \\
= & e^{i x}-3 i e^{x} \frac{t^{\alpha}}{\Gamma(\alpha+1)}+3^{2} i^{2} e^{i x} \frac{t^{2 \alpha}}{\Gamma(2 \alpha+1)} \\
& -63 i^{3} e^{i x} \frac{t^{3 \alpha}}{\Gamma(3 \alpha+1)}+18 i^{3} e^{i x} \frac{t^{3 \alpha}}{\Gamma(3 \alpha+1)} \\
& \frac{\Gamma(2 \alpha+1)}{\Gamma(\alpha+1)^{2}}+\ldots
\end{aligned}
$$

then

$$
\begin{aligned}
u=\sum_{i=0}^{3} u_{i}= & e^{i x}\left(1-3 i \frac{t^{2 \alpha}}{\Gamma(2 \alpha+1)}+\left(3 i^{2}\right) \frac{t^{2 \alpha}}{\Gamma(2 \alpha+1)}\right. \\
& -(3 i)^{3} \frac{t^{3 \alpha}}{\Gamma(3 \alpha+1)}+\ldots
\end{aligned}
$$

In closed form, we obtain the solution:

$$
u=e^{i x}\left(\sum_{n=0}^{\infty}(-3 i)^{n} \frac{t^{n \alpha}}{\Gamma(n \alpha+1)}\right)
$$

The special case $\alpha=1$, we obtain that $u(x, t)=e^{i x}-3 i e^{i x} t+(3 i)^{2} e^{i x} \frac{t^{2}}{2 !}$

$$
-(3 i)^{3} e^{i x} \frac{t^{3}}{3 !}+\ldots=e^{i(x-3 t)} .
$$

Which is the same exact solution as obtained by PM.

It is clear that the result obtained by HPM in (70) is the same result as obtained by PM in (63).

In Fig. (3), we have plotted the approximate solution for nonlinear Schrödinger equation (55) for the cases a) $\alpha=0.25$, b) $\alpha=0.5$, c) $\alpha=0.75$, d) $\alpha=1$, and e) the corresponding exact solution.

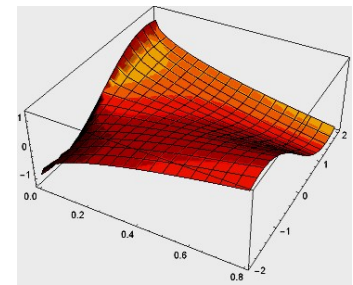

(a) $\alpha=0.25$

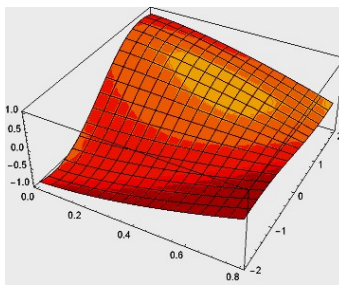

(c) $\alpha=0.75$

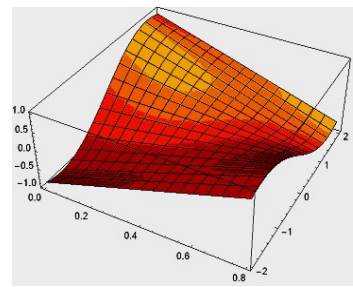

(b) $\alpha=0.50$

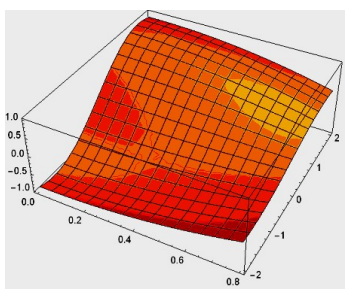

(d) $\alpha=1.0$

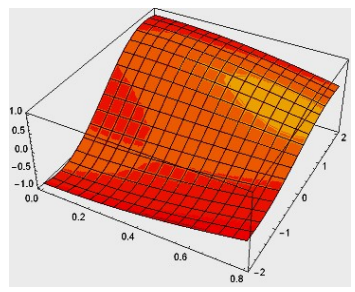

(e) Exact solution

Fig. 3. The surface shows the approximate solution for (59) for various values at $\alpha$ with the corresponding exact solution.

Example 4. We consider the nonlinear timefractional Schrödinger equation

$i D_{t}^{\alpha}=-\frac{1}{2} u_{x x}+u \cos ^{2} x+\left|u^{2}\right| u$,

with the initial condition 
$u(x, 0)=\sin x$

The exact solution is

$u(x, t)=e^{\frac{-3 i t}{2} \sin x}$.

We rewrite the equation (71) in the form

$D_{t}^{\alpha} u=\frac{1}{2} i u_{x x}-i u \cos ^{2} x-i u^{2} \bar{u}$.

In the equation we have $\beta=\frac{1}{2}, \gamma=-1$, and $v(x)=-\cos ^{2} x$.

1- By PM: we construct the following iteration formula for Picard:

$$
\begin{gathered}
u_{j+1}=u_{0}+I_{t}^{\alpha}\left[\frac{1}{2} i u_{j_{x x}}-i u_{j} \cos ^{2} x-i u_{j}^{2} \bar{u}_{j}\right] \\
j \geq 0
\end{gathered}
$$

Therefore, we can obtain the following first few terms of required solution

$$
\begin{aligned}
u_{0}= & \sin x \\
u_{1}= & \sin x-\frac{3}{2} i \sin x \frac{t^{\alpha}}{\Gamma(\alpha+1)} \\
u_{2}= & \sin x-\frac{3}{2} i \sin \frac{t^{\alpha}}{\Gamma(\alpha+1)}+\frac{9}{4} i^{2} \sin x \frac{t^{2 \alpha}}{\Gamma(2 \alpha+1)}-\ldots \\
u_{3}= & \sin x-\frac{3}{2} i \sin \frac{t^{\alpha}}{\Gamma(\alpha+1)}+\frac{9}{4} i^{2} \sin x \frac{t^{2 \alpha}}{\Gamma(2 \alpha+1)} \\
& -\frac{27}{8} i^{3} \frac{t^{3 \alpha}}{\Gamma(3 \alpha+1)}+\ldots
\end{aligned}
$$

then

$$
\begin{aligned}
u= & \sin x\left(1-\frac{3}{2} \frac{i t^{\alpha}}{\Gamma(\alpha+1)}+\frac{9}{4} i^{2} \frac{t^{2 \alpha}}{\Gamma(2 \alpha+1)}\right. \\
& -\frac{27}{8} i^{3} \frac{t^{3 \alpha}}{\Gamma(3 \alpha+1)}+\ldots .
\end{aligned}
$$

In closed form, we obtain the solution:

$$
u=\sin x\left(\sum_{n=0}^{\infty}\left(-\frac{3 i}{2}\right)^{n} \frac{t^{n \alpha}}{\Gamma(n \alpha+1)}\right) .
$$

$$
\begin{aligned}
u(x, t) & =\sin x\left(1-\frac{3}{2} i t+\left(\frac{3 i t}{2}\right)^{2} \frac{1}{2 !}-\left(\frac{3 i t}{2}\right)^{3} \frac{1}{3 !}+\ldots\right) \\
& =e^{-\frac{2 i t}{2}} \sin x
\end{aligned}
$$

which is an exact solution for (72).

2- By HPM: the homotopy for (72) becomes

$$
D_{t}^{\alpha}=i p\left[\frac{1}{2} u_{x x}-\cos ^{2} x u-u^{2} \bar{u}\right] \text {, }
$$

we suppose that the solution of the problem (74) is in the form

$$
u=u_{0}+p u_{1}+p^{2} u_{2}+p^{3} u_{3}+\ldots,
$$

substituting (76) into (77), we obtain:

$$
\begin{aligned}
& D_{t}^{\alpha}\left[u_{0}+p u_{1}+p^{2} u_{2}+p^{3} u_{3}+\ldots\right]=i p\left[\frac { 1 } { 2 } \left[u_{0}+p u_{1}+p^{2} u_{2}\right.\right. \\
& \left.\quad+p^{3} u_{3}+\ldots\right]_{x x}-\cos ^{2} x\left[u_{0}+p u_{1}+p^{2} u_{2}+p^{3} u_{3}+\ldots\right] \\
& \quad-\left[u_{0}+p u_{1}+p^{2} u_{2}+p^{3} u_{3}+\ldots\right]^{2}\left[\overline{u_{0}}+p \bar{u}_{1}+p^{2} \bar{u}_{2}\right. \\
& \left.\left.+p^{3} \bar{u}_{3}+\ldots\right]\right]
\end{aligned}
$$

Equating the terms of equal powers $p$ in both sides of the above equation, we obtain:

$$
\begin{array}{rlr}
p^{0}: D_{t}^{\alpha} u_{0}= & 0, & u_{0}(x, 0)=\sin x, \\
p^{1}: D_{t}^{\alpha} u_{1}= & \frac{1}{2} i u_{0_{x x}}-i \cos ^{2} x u_{0}-i u_{0}^{2} \bar{u}_{0}, & u_{1}(x, 0)=0 \\
p^{2}: D_{t}^{\alpha} u_{2}= & \frac{1}{2} i u_{1_{x x}}-i \cos ^{2} x u_{1}-i u_{0}^{2} \bar{u}_{1} & \\
& -2 i u_{0} u_{1} \bar{u}_{0}, & \\
p^{3}: D_{t}^{\alpha} u_{3}= & \frac{1}{2} i u_{2_{x x}}-i \cos ^{2} x u_{2}-i u_{0}^{2} \bar{u}_{2}-i \bar{u}_{0}\left(u_{1}^{2}\right. \\
& \left.+2 u_{0} u_{2}\right)-2 i u_{0} u_{1} \bar{u}_{1} & \\
\vdots & & u_{3}(x, 0)=0
\end{array}
$$

Solving the above set of equation in (79), we obtain

For the special case $\alpha=1$, we obtain that: 


$$
\left.\begin{array}{rl}
u_{0}= & \sin x, \\
u_{1}= & -\frac{3}{2} i \sin x \frac{t^{\alpha}}{\Gamma(\alpha+1)}, \\
u_{2}= & -\frac{9}{4} i^{2} \sin x \frac{t^{2 \alpha}}{\Gamma(2 \alpha+1)}, \\
u_{3}= & -\frac{9}{8} i^{3} \sin x \frac{t^{3 \alpha}}{\Gamma(3 \alpha+1)}-\frac{9}{4} i^{3} \sin ^{2} x \sin x \frac{t^{3 \alpha}}{\Gamma(3 \alpha+1)} \\
& -\frac{27}{4} i^{3} \sin ^{3} x \frac{t^{3 \alpha}}{\Gamma(3 \alpha+1)}+\frac{9}{4} i^{3} \sin ^{3} x \frac{t^{3 \alpha}}{\Gamma(3 \alpha+1)} \frac{\Gamma(2 \alpha+1)}{\Gamma(\alpha+1)^{2}}
\end{array}\right\}
$$

So the solution will be

$$
\begin{aligned}
u(x, t)= & u_{0}+u_{1}+u_{2}+u_{3}+\ldots \\
= & \sin x-\frac{3}{2} i \sin x \frac{t^{\alpha}}{\Gamma(\alpha+1)}+\frac{9}{4} i^{2} \sin x \\
& \frac{t^{2 \alpha}}{\Gamma(2 \alpha+1)}-\frac{9}{8} i^{3} \sin x \frac{t^{3 \alpha}}{\Gamma(3 \alpha+1)} \\
& -\frac{9}{4} i^{3} \cos ^{2} x \sin x \frac{t^{3 \alpha}}{\Gamma(3 \alpha+1)}-\frac{27}{4} i^{3} \sin ^{3} x \\
& \frac{t^{3 \alpha}}{\Gamma(3 \alpha+1)}+\frac{9}{4} i^{3} \sin ^{3} x \frac{t^{3 \alpha}}{\Gamma(3 \alpha+1)} \frac{\Gamma(2 \alpha+1)}{\Gamma(\alpha+1)^{2}}+\ldots,
\end{aligned}
$$

then,

$$
\begin{aligned}
u=\sum_{i=0}^{3} u_{i}= & \sin x\left(1-\frac{3}{2} \frac{t^{\alpha}}{\Gamma(\alpha+1)}+\frac{9}{4} i^{2} \frac{t^{2 \alpha}}{\Gamma(2 \alpha+1)}\right. \\
& \left.-\frac{27}{8} i^{3} \frac{t^{3 \alpha}}{\Gamma(3 \alpha+1)}+\ldots\right) .
\end{aligned}
$$

In closed form, we obtain the solution

$$
u=\sin x\left(\sum_{n=0}^{\infty}\left(-\frac{3}{2} i\right)^{n} \frac{t^{n \alpha}}{\Gamma(n \alpha+1)}\right) .
$$

For the special case $\alpha=1$, we obtain that

$$
\begin{aligned}
u(x, t)= & \sin x-\frac{3 i t}{2} \sin x+\frac{\left(\frac{3}{2} i t\right)^{2}}{2 !} \sin x \\
& -\frac{\left(\frac{3}{2} i t\right)^{3}}{3 !} \sin x+\ldots
\end{aligned}
$$

$$
=e^{-\frac{3 i t}{2}} \sin x,
$$

which is the same exact solution as obtained by PM.

It is clear that the result obtained by HPM in (82) is the same result as obtained by PM in (75)

In Fig. (4), we have plotted the approximate solution for the nonlinear Schrödinger equation (77) for cases a) $\alpha=0.25$, b) $\alpha=0.5$, c) $\alpha=0.75$, d) $\alpha=1$, and e) the corresponding exact solution.

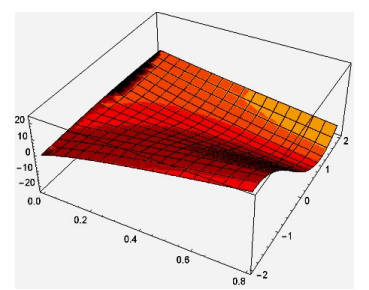

(a) $\alpha=0.25$

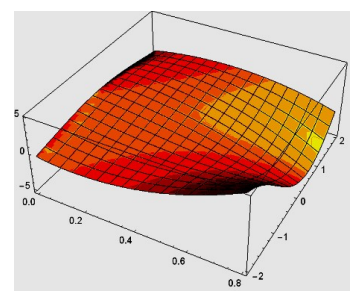

(c) $\alpha=0.75$

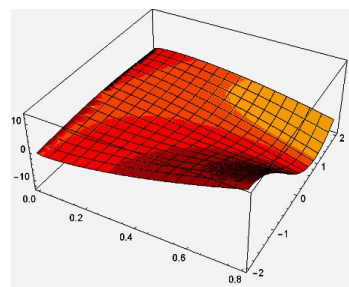

(b) $\alpha=0.50$

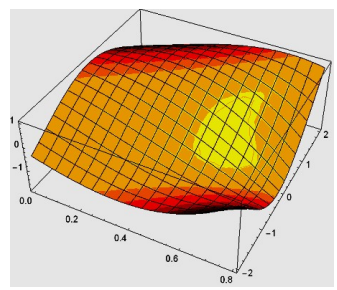

(d) $\alpha=1.0$

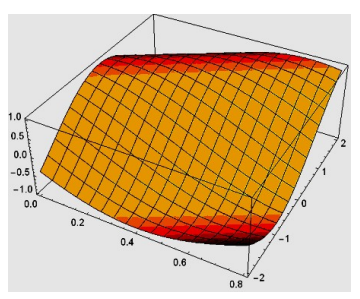

(e) Exact solution

Fig. 4. The surface shows the approximate solution for (72) for various values of $\alpha$ with the corresponding exact solution.

\section{References}

[1] Z. Ayati, J. Biazar, and S. Ebrahimi, A new homotopy perturbation method for solving linear and nonlinear Schrödinger equations, Journal of Interpolation and Approximation in Scientific Computing, 2014, (2014), 1-8. 
[2] B. N. N. Achar, B. T. Yale, and J. W. Hanneken, Time Fractional Schrödinger EquationRevisited, Advances in Mathematical Physics, (2013), 1-11.

[3] Z. Bai, S. Zhang, S. Sun, and C. Yin, Monotone Iterative Method for Fractional Differential Equations, Electronic Journal of Differential Equations, 2016(6), (2016), 1-8.

[4] A. Bibi, A. Kamran, U. Hayat and S. T. MohyDin, New iterative method for time-fractional Schrödinger equations, World Journal of Modeling and Simulation, vol. 9, (2013), no. 2, pp. 89-95.

[5] V. Daftardar-Gejji, and S. Bhalekar, Solving fractional boundary value problems with dirichlet boundary conditions using a new iterative method. Computers and mathematics with Applications, (2010), 1801-1809.

[6] A. El-Ajou, Z. Odibat, S. Momani, and A. Alwneh, Construction of Analytical Solutions to Fractional Differential Equations Using Homotopy Analysis Method, IAENG International Journal of Applied Mathematics, 40 (2), (2010).

[7] M. H. Eljaily1, and T. M. Elzaki, Solution of Linear and Nonlinear Schrodinger Equations by Combine Elzaki Transform and Homotopy Perturbation Method American Journal of Theoretical and Applied Statistics, 4(6), (2015), 534-538.

[8] A. Goyal, Solitons: An Introduction. Department of Physics, Panjab University, Chandigraph.

[9] A. A. Hemeda, Modified homotopy perturbation method for solving fractional differential equations. Journal of Applied Mathematics, (2014).
[10] H. Jafari, H. Tajadodi, and D. Baleanu, A Modified Variational Iteration Method for Solving Fractional Riccati Differential Equation by Adomian Polynomials, Fractional Calculus and Applied Analysis, vol. 16, no. 1, (2013), 109-122.

[11] T. Kevkić, V. Stojanović, and D. Randjelović, Application of Homotopy Perturbation Method in Solving Coupled Schrödinger and Poisson Equation in Accumulation Layer, Romanian Journal of Physics, 62, 122, (2017), 1-13.

[12] N. A. Khan, A. Ara, and N. A. Khan, Fractional-order Riccati differential equation: Analytical approximation and numerical results, Advances in Difference Equations, (2013), 1-16.

[13] R. Lyons, A S. Vatsala, and R. A. Chiquet, Picard's Iterative Method for Caputo Fractional Differential Equations with Numerical Results, Mathematics5, 65, (2017), doi:10.3390/math5040065, http://www.mdpi.com/journal/mathematics.

[14] K. Maleknejada, and L. Torkzadeha, Hybrid Functions Approach for the Fractional Riccati Differential Equation Published by Faculty of Sciences and Mathematics, University of Niš, Serbia, 30(9), (2016), 2453-2463, http://www.pmf.ni.ac.rs/filomat.

[15] M. Merdan, On the Solutions Fractional Riccati Differential Equation with Modified Riemann-Liouville Derivative, International Journal of Differential Equations, (2012), 117.

[16] M. M. Mousaa, and A. Kaltayevb, Homotopy Perturbation Method for Solving Nonlinear Differential- Difference Equations Verlag der Zeitschrift für Naturforschung, 65a, (2010), 511- 517, Tubingen, http://znaturforsch.com. 
[17] A. Mohebbi, On the split-step method for the solution of nonlinear Schrödinger equation with the Riesz space fractional derivative, Computational Methods for Differential Equations, vol. 4, no. (1), (2016), pp. 54-69.

[18] A. A. Nahla, and A. A. Hemeda, Picard Iteration and Padé Approximations for Stiff Fractional Point Kinetics Equations, Applied Mathematics and Computation, 293, (2017), $72-80$.

[19] K. Nisse, and L. Nisse, An Iterative Method for Solving a Class of Fractional Functional Differential Equations with Maximal Mathematics, 6, 2, (2018), www.mdpi.com/journal/mathematics.

[20] P. Rahimkani, Y. Ordokhani, and E. Babolian, Application of fractional-order Bernoulli functions for solving fractional Riccati differential equation, Int. J. Nonlinear Anal. Appl., 8:(2), (2017), 277-292.

[21] R. Rozmej, and Bandrowski, On fractional Schrödinger equation, Computational methods in Science and technology, 16(2), (2010), 191194.

[22] B. K. Singh, and P. Kumar, Homotopy perturbation transform method for solving fractional partial differential equations with proportional delay, Math. NA, (2016), 1-15.

[23] S. Tomasiello, and J. E. Macias-Diaz, Note on a Picard-Like Method for Caputo Fuzzy Fractional Differential Equations, Applied Mathematics and Information Sciences, 11(1), (2017), 281-287.

[24] A. Yildirim, S. Mohyud-Din, Analytical approach to space and time fractional burger's equations. Chinese Physics Letters, 27(9), (2010): 090501. 